\title{
Bearing diagnostics under strong electromagnetic interference based on Integrated Spectral Coherence
}

\author{
Alexandre Mauricio ${ }^{\mathrm{a}, \mathrm{b}}$, Junyu Qia ${ }^{\mathrm{a}, \mathrm{b}}$, Wade A. Smith ${ }^{\mathrm{c}}$, Mathieu Sarazin ${ }^{\mathrm{d}}$, \\ Robert B. Randall ${ }^{\mathrm{c}}$, Karl Janssens ${ }^{\mathrm{d}}$, Konstantinos Gryllias ${ }^{\mathrm{a}, \mathrm{b}, *}$ \\ ${ }^{a}$ Faculty of Engineering Science, Department of Mechanical Engineering, Division PMA, \\ KU Leuven \\ Celestijnenlaan 300, Box 2420, 3001 Leuven, Belgium \\ ${ }^{b}$ Dynamics of Mechanical and Mechatronics Systems, Flanders Make, Belgium \\ ${ }^{c}$ School of Mechanical and Manufacturing Engineering, University of New South Wales \\ Sydney, NSW 2052, Australia \\ ${ }^{d}$ Siemens Industry Software NV, Interleuvenlaan 68, 3001 Leuven, Belgium
}

\begin{abstract}
Rolling element bearing fault diagnostics has been a topic of intensive research. As the rolling element bearings are a critical component of rotating machinery, failure of these components results in the breakdown of the mechanical structure in which they operate. The correct detection of incipient faults on the bearings can reduce the production cost by allowing maintenance engineers to schedule a replacement at the most convenient time. Envelope analysis is a widespread method in bearing diagnostics, where the frequency band that carries the fault information is detected and amplitude demodulated to obtain the envelope, whose spectrum reveals the repetition frequencies of the particular fault. It is often used along with Fast Kurtogram, which selects this band based on the kurtosis level. However, this methodology shows difficulty in selecting the correct band on complex signals. Signals contaminated with Electro-Magnetic Interference (EMI) are one such example where Envelope Analysis based on the Fast Kurtogram (FK) often fails to diagnose bearing faults. EMI is often present in mechanisms whose motor is controlled by a Variable-Frequency Drive (VFD), and shows a behaviour similar to that of bearing faults, and thus increases the
\end{abstract}

\footnotetext{
${ }^{*}$ Corresponding Author

Email address: konstantinos.gryllias@kuleuven.be (Konstantinos Gryllias )
}

Preprint submitted to Mechanical Systems and Signal Processing 
complexity in bearing fault detection. The Cyclic Spectral Coherence (CSCoh) is used in this paper as a substitute methodology for bearing diagnostics, as it shows better performance in modelling the bearing fault signals, due to being a powerful tool in the analysis of second order cyclostationary signals. From the output of the CSCoh it is possible to detect each of the possible the fault frequencies and their corresponding carrier frequencies. Integration of the CSCoh over the full spectral frequency results on the Enhanced Envelope Spectrum (EES), which is equivalent to the Squared Envelope Spectrum (SES) from Envelope Analysis. Furthermore, integration on a set band over the spectral frequency of the CSCoh results on the Improved Envelope Spectrum (IES) which is equivalent to band-pass filtering the signal before obtaining the SES. This paper presents two methods that allow the selection and integration of the optimal bands on the CSCoh which enhance the fault frequencies of bearing faults without human analysis. The method is applied to experimental data contaminated with EMI and shows higher performance in detecting a faulty bearing when compared to the EES and Envelope Analysis.

Keywords: Cyclic Spectral coherence, Condition monitoring, Bearing diagnostics, Electromagnetic interference

\section{Introduction}

Condition monitoring of rotating machinery is often performed based signals. These signals measured from real application always have some inherent noise that difficults the health monitoring of the machinery. Electromagnetic interference (EMI) is one such common and unwanted component captured by 6 vibration sensors (accelerometers) and microphones. This type noise is generally emitted, conducted or radiated from the Variable Frequency Drives (VFD) that control the motor shaft speed, providing power to various rotating machinery. Therefore it is important to process the signals taking into account the effect of EMI. Therefore, insight on the nature of the noise is necessary when signals are contaminated with EMI. Smith et. al. 1, 2, explained how the unwanted 
signal can be simulated using a phenomenological model, and why it is harmful to the machinery health diagnosis. The simulation of EMI results in the same broadband impulsivity that real measurements with EMI contamination present. The nature of this signal is particularly problematic for the diagnostics of rolling element bearings, as both EMI polluted signals and signals emitted by defected rolling element bearings present an impulsive behavior. In other words, both the damage and unwanted phenomena have a similar signature. Common signal processing methods use for fault detection tend to exploit and/or enhance the impulsiveness of signals. As both the fault and the EMI contamination present a periodic impulsive nature, the methods may enhance the EMI instead of the fault signature or detect the modulations of the EMI effect instead of the modulations caused by the bearing fault [3].

Envelope Analysis has dominated the field as a pre-processing tool in bearing diagnostics. The signal is demodulated, often using the Hilbert Transform, and the envelope spectrum of the demodulated signals is then analysed. The resulting spectrum from which the analysis is obtained is commonly known as the Squared Envelope Spectrum (SES). In order to enhance the detection, the signal is usually filtered around an excited frequency band (resonance) so containing the fault information and where the Signal-to-Noise Ratio (SNR) is high. The frequency band selection can be done by human expertise or by methodologies which select the filter band automatically by optimising a specific criteria, such as the spectral kurtosis (Fast Kurtogram) [4, 5] and the peak energy criterion [6]. Following the methodology of the Fast Kurtogram (FK), a series of filters with different bandwidths and center frequencies is applied on 36 the signal, following a $1 / 3$ binary tree. The kurtosis level of the filtered signal is calculated for each applied filter. The parameters of the filter resulting in the maximum kurtosis are selected [7]. The method shows good fault diagnosis results [8] as the signal generated by the bearing faults tends to be rather impulsive and the selection of the filter based on the maximisation of kurtosis, further enhances the impulsiveness of the filtered signal. On the other hand, ${ }_{42}$ the presence of impulsive noise or of outliers may reduce the performance of the 
method [9]. Using a experimental case based on a planetary gearbox, Smith et. al. 2, 3] explained that the FK failed to select the optimal filter parameters because the allowed bandwidth was too wide. They concluded that by selecting a narrow and constant bandwidth, better filter parameters could be selected. A methodology similar to FK, entitled Optimized Spectral Kurtosis (OSK) was 48 then proposed, introducing a series of filters with a constant narrow bandwidth instead of a variable one. The method was successfully applied on vibration signals strongly contaminated by EMI captured on a planetary gearbox with a defective bearing positioned on one of the planets [2].

Cyclic Spectral Correlation (CSC) has been recently introduced as a powerful tool in the area of condition monitoring. It has been successfully applied in a number of cases and presents good performance on the diagnostics of damaged rolling element bearings [10, 11]. An enhanced version of the CSC, the Cyclic Spectral Coherence (CSCoh), appears to be more sensitive on the exploitation of the level of cyclostationarity of signals [12]. The method generates a two dimensional map in which the signal can be described in two axes: the spectral frequency axis, which contains the excited natural frequency components; and the cyclic frequency axis, which contains the characteristic modulation frequency component. When this 2D matrix is integrated along the spectral frequency it results in a spectrum of the demodulated signal from which the fault frequencies can be detected. Two variant procedures can be extracted from this procedure. The simplest one is equivalent to the demodulated spectrum (SES), consisting on the integration over the full spectral frequency (from 0 ${ }_{66}$ to the Nyquist frequency). This resulting spectrum was coined the Enhanced Envelope Spectrum (EES). The other procedure is the integration of a specific band, instead of integration over the full band. This spectrum was coined the Improved Envelope Spectrum (IES) and is equivalent to the demodulated spectrum (SES) of the band-pass filtered signal.

In this paper two novel methodologies are proposed focusing towards the 72 automated rolling element bearing fault detection in rotating machinery under EMI influence. The methodologies detect the optimal band of frequencies 
to be integrated on the CSCoh, leading to the Improved Envelope Spectrum (IES) with the bearing damage information. The approaches have been tested, validated and evaluated on an experimental case of a planetary gearbox. Inner and outer race bearing faults located at one of the planets of the planetary gearbox operating under the influence of strong EMI are analysed and the performance of the methodologies are compared to state of the art diagnostic tools.

The paper is organised as follows. The principle of the EMI generation and bearing fault detection are introduced in Section 2 2 Different signal processing methods for bearing diagnostics, including the CSC upon which the new methods are based on, are reviewed in Section 3 . In Section 4 the two new methods are presented in detail. Furthermore the experimental setup is introduced in Section 5. The methodologies are applied on Section 6 and the new methods are evaluated and compared with the classical CSC, OSK and the classical Envelope Analysis. Finally some conclusions are drawn in Section 7 .

\section{Bearing fault signals with electromagnetic interference contamination}

When a localized defect in one surface of a rolling element bearing strikes another surface, it produces an impulse which excites structural resonances in the bearing and in the machine. As the bearing rotates, these impulses will occur with a set period determined uniquely by the geometry of the bearing and the location of the defect. McFadden and Smith [13] proposed a model 96 of the phenomenon and explain why the Envelope Analysis allows for the successful detection of faults. The impacts caused by the generation of a bearing fault lead to the excitation of natural frequencies of the bearing and/or the structure, which appear usually at high frequencies. These excited frequencies are modulated by the characteristic frequency of the bearing fault, and are denominated the carriers. Demodulation methods are shown to be important in order to extract the characteristic frequencies of the defect. In a first step, 
signals are commonly filtered around an excited frequency band where the SNR is higher. The selection of the optimum frequency band (central frequency and bandwidth) can be done by the vibration analyst or by an automated procedure based on the optimisation of a criterion, such as the Kurtogram which selects the optimum frequency band by maximising the spectral kurtosis. The most common applied form is the Fast Kurtogram (FK) as it is seen as a fast preprocessing technique whose code is present in most of the signal processing solvers in the market.

Electromagnetic interference signal is defined as any unwanted signal (electrical noise) that is captured by the sensors due to induction or conduction to the electronics equipment. Most common causes of EMI generation occur via a Variable Frequency Drive (VFD) controlling a motor. The VFD converts an $\mathrm{AC}$ voltage signal to an $\mathrm{AC}$ output of a different voltage and frequency in order to generate the speed control of the motor shaft. The most usual output signals for a VFD are the Pulse-Width Modulated (PWM) output signals [2]. PWM signals are pulse train signals modulated in frequency by the speed signal. The integration of the PWM results in a speed signal with higher frequency components corresponding to the switch frequency of the VFD. PWM signals can be generated (along with the resultant EMI noise) by comparing a sinusoid at the reference speed with a triangular waveform at the switching frequency of the drive. When the speed profile is greater than the triangular form the PWM will be null while in the opposite case when it is smaller, the PWM will be unitary. The EMI that results from a PWM signal arises from current spikes (high frequency transients in the electrical system) that occur at each step in the PWM signal (sometimes only in the negative steps, as described in [2] ). This generation process is described in figure 1. The spectrum of an EMI signal usually presents harmonics of the switching frequency of the PWM (usually between 2 and $16 \mathrm{kHz}$ ) modulated by harmonics of a frequency which corresponds to the pseudo-line frequency (depends on the number of poles and the slip of the motor, but is commonly a integer multiple of the motor shaft speed) and possibly by harmonics of the line frequency $\left(f_{L}=50 \mathrm{~Hz}\right.$, in most 
countries).

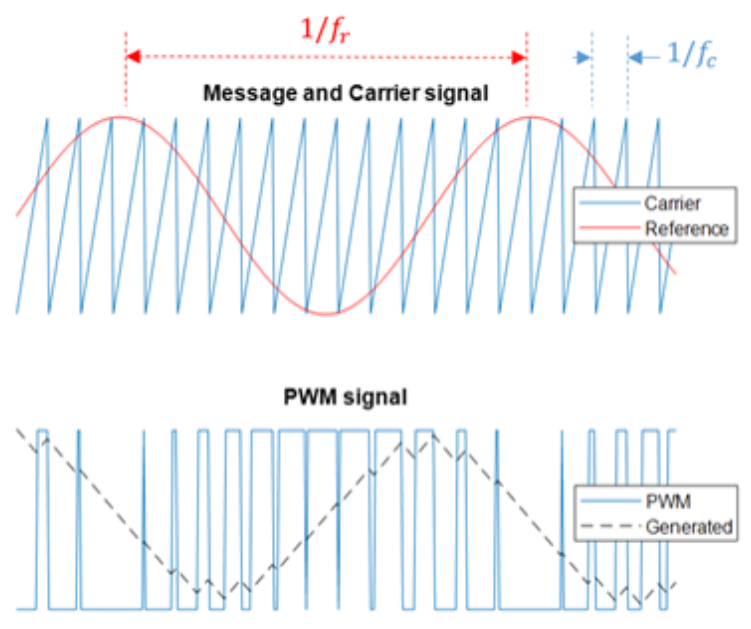

Figure 1: Generation process of a PWM signal.

Therefore it should be highlighted that both defective bearing signals and EMI polluted signals present similar behavior, containing periodicity and impulsiveness. This can be demonstrated in the example signal presented in figure 2a, which is composed of an EMI time signal and a simulated bearing signal, generated by a simple rolling element bearing model containing a defect in the inner race. The signal-to-EMI ratio is quite high in this case (in other words, the presence of EMI on the signal is quite weak compared to the bearing signature), and the impulsive nature of both signals is evident. Moreover the corresponding spectrum of the two signals is presented in $2 \mathrm{~b}$. Two harmonics of the switch frequency, which are selected to be equal to 5 and $10 \mathrm{kHz}$, are seen to be present and both are modulated by the motor pseudo-line frequency. Additionally an excited frequency band is present around the bearing natural frequency $f_{n}$, which has been set at $7.7 \mathrm{kHz}$, is modulated by the characteristic inner race defect frequency (BPFI). The impulsiveness and the periodicity of the EMI signal, even when the Signal-to-EMI ratio is high, may mislead classical diagnostic tools into selecting the harmonics of the switch frequency as the 
demodulation band, instead of the excited natural frequency. As a result, the spectrum of the envelope signal will be dominated by harmonics of the motor speed and/or of the line frequency and not by the characteristic frequency of the defect, leading to an incorrect diagnosis.

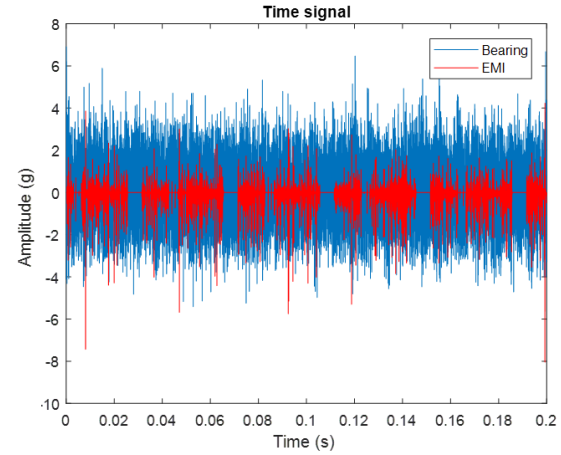

(a) Time signals.

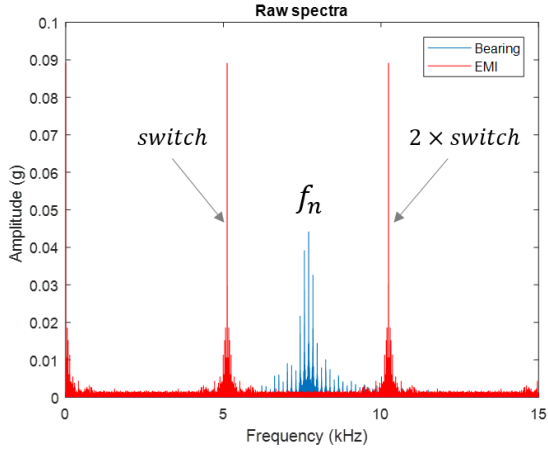

(b) Spectra.

Figure 2: Simulated inner race fault and EMI signals.

To provide insight into this phenomenon, a simple example on two simulated

signals is exposed in figure 3 the first signal contains the signature of an inner race damaged bearing without EMI contamination; the second signal is the same as the previous, plus a strong EMI contamination signature. The envelope analysis on the first simulated signal (without EMI) exhibits clear peaks at the BPFI modulated by the shaft frequency. This is due to the Kurtogram selecting a narrow band around the resonant frequencies. On the other hand, the presence of EMI on the second simulated signal resulted in the selection of a different band of demodulation, therefore resulting Squared Envelope Spectrum exhibiting only peaks at the shaft frequency, which is the pseudo-line frequency.

\section{Signal processing methods for rolling element bearing diagnostics}

Advanced signal processing methods are usually applied on vibration signals in an effort to detect impacts related to bearing defects, increase SNR and further demodulate the signal, in order to accurately detect the presence of a 

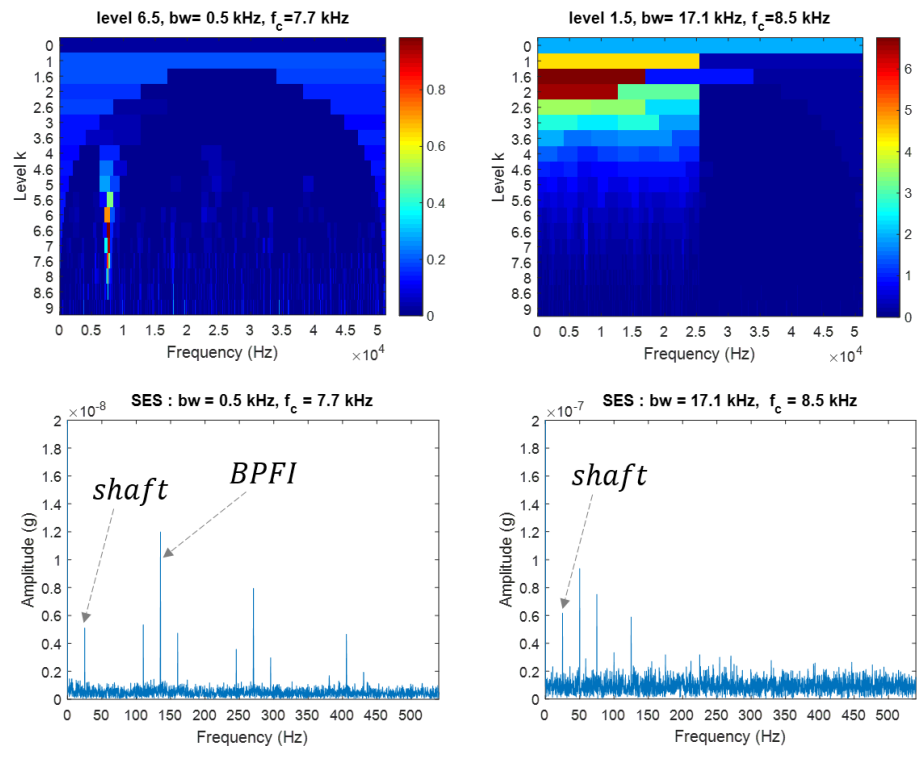

(a) Without EMI.

(b) With EMI.

Figure 3: Fast Kurtograms and Squared Envelope Spectra for simulated bearing inner race fault signals with and without EMI.

bearing defect, trying in parallel to simplify the diagnosis procedure. As it was previously commented, classical diagnostic methodologies may fail to detect accurately a rolling element fault under the presence of strong EMI. In this section three well known methods are presented, including the classical Squared Envelope Spectrum (SES) [5],the Optimized Spectral Kurtosis (OSK) 2] and the Enhanced Envelope Spectrum (EES) [5]. The second one has been recently proposed by Smith et. al. as a bearing diagnostics method to overcome the EMI influence and will be used later as a reference.

\subsection{Squared Envelope Spectrum (SES) by Fast Kurtogram (FK) filter selection}

The Squared Envelope Spectrum (SES) is obtained as the absolute of the Hilbert transformed signal. The methodology can be applied on the raw signal, but without further signal processing, the corresponding diagnostic results are often poor. Usually the raw signal is firstly filtered around a frequency band excited by the defect, thus increasing the SNR and enhancing the harmonics of 


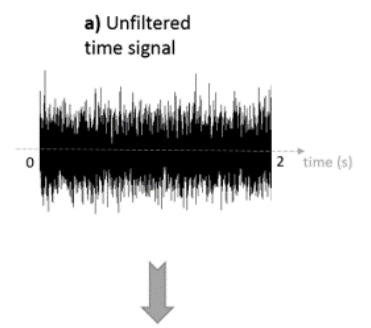

b) Spectrum

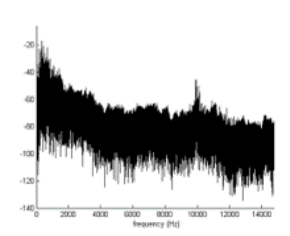

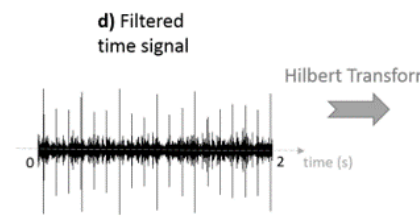

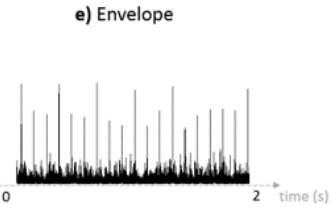

$$
\begin{aligned}
& \text { Band-Pass } \\
& \text { Filtering }
\end{aligned}
$$

c) Zoomed Spectrum

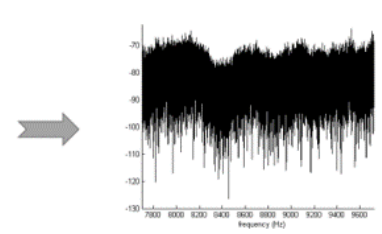

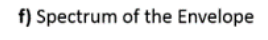

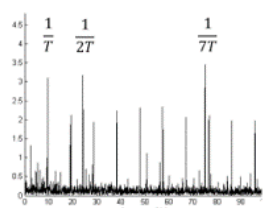

Figure 4: Envelope analysis procedure.

the fault frequency component in the SES. Discrete/Random Separation-type operations do not remove successfully the EMI from the signal, as both the bearings and EMI signatures are not deterministic [2].

The described procedure known as Envelope Analysis is depicted in figure 4 In a first step, the spectrum of the unfiltered signal is formed and analyzed in order to select the parameters of a band-pass filter. A number of methodologies have been presented in the past in order to optimally select the central frequency and the bandwidth of the filter. In this paper, the Fast Kurtogram (FK) is used in order to estimate the parameters of the band-pass filter. This process maximises the spectral kurtosis for a series of filters, and thus automates the diagnostic procedure without any human intervention that would be needed otherwise.

\subsection{Optimized Spectral Kurtosis (OSK)}

The Optimized Spectral Kurtosis (OSK) method also includes the application of a series of filters on the raw signal. The main difference when compared to the FK lies on the bandwidth of the applied filters, which is kept constant while the central frequency of the filter scans the full frequency range. The user selects the bandwidth (based on knowledge of the likely fault 
frequencies) and the step/increment of the central frequency scanning (based on allowable computational cost). At each step, the raw signal is filtered and the kurtosis of the filtered signal is estimated. Finally the parameters of the filter are selected based on the maximization of the kurtosis, following the exact same procedure as with FK.

Smith et. al. applied the method on various types of vibration signals of bearings presenting a defect and operating under strong EMI [2] and concluded that a filter with a small bandwidth should be chosen based on engineering experience, between three and eight times the characteristic fault frequency. The method showed good diagnostic performance on planetary gearbox signals under the presence of strong EMI.

\subsection{Enhanced Envelope Spectrum (EES) extracted from the Cyclic Spectral Coherence (CSCoh)}

Most mechanical rotating parts with stationary periodic components can be well represented by Fourier transform based methods, such as the WignerVille spectrum. However, most components, bearings in particular, show a cyclostationary signal carrying hidden periodicities in its structure [14]. Bearing signals can be described as second-order cyclostationary signals presenting a periodic autocorrelation, or, in other words, as signals having the same ensemble expected value of the autocorrelation at a set time-lag $\tau$. A second-order cyclostationary signal can be defined as a $T$-periodic autocorrelation function meeting the condition described by equation 1

$$
R_{x}\left(t_{n}, \tau\right)=R_{x}\left(t_{n}+T, \tau\right)=\mathbb{E}\left\{x\left(t_{n}\right) x\left(t_{n}-\tau\right)^{*}\right\}
$$

where $R_{x}$ is the autocorrelation function, $\mathbb{E}$ stands for the ensemble average operator, $x()$ for the signal, $t_{n}$ for a periodic function of time with period $T$.

The Cyclic Spectral Correlation (CSC) has been proposed as a powerful tool for detection and characterization of cyclostationarity signals in engineering applications. The method exploits well the unknown cyclic frequencies which modulate the signal, particularly in the case of localized faults in rolling 
element bearings, where the fault frequency has some random variation [5]. The statistical descriptor of second-order cyclostationarity by the CSC can be described by equation 2 .

$$
S_{x}(\alpha, f)=\lim _{W \rightarrow \infty} \frac{1}{W} \mathbb{E}\left\{X_{w}^{*}(f) X_{w}\left(f+\alpha_{t}\right)\right\}
$$

Where $X_{w}(f)=\int_{-W / 2}^{+W / 2} x(t) e^{j 2 \pi t f} d t$ is the Fourier transform of the signal $x(t)$ over a time interval $W, f$ is the spectral frequency dual with time, and $\alpha$ is the cyclic frequency dual with time-lag. The expression measures the correlation level between two frequency components of the signal at $f$ and $f+\alpha_{t}$ [14].

The CSC results in a 2-D map which declares the modulation frequencies in the $\alpha$ axis and their frequency content $f$. Antoni [11] further suggests the Cyclic Spectral Coherence (CSCoh), which is calculated based on the CSC as shown in equation 3

$$
\gamma_{x}(\alpha, f)=\frac{S_{x}(\alpha, f)}{\sqrt{S_{x}(0, f-\alpha / 2) S_{x}(0, f+\alpha / 2)}}
$$

where $S_{x}(\alpha, f)$ is the spectral correlation.

The CSCoh may also be interpreted as the CSC of the whitened signal, which tends to equalize regions with very different energy levels and thus to magnify weak cyclostationary signals, as Antoni et. al. suggest [11. They also propose the Enhanced Envelope Spectrum (EES) where the CSCoh is integrated over the full band of spectral frequencies $\left[0 ; f_{s} / 2\right]$, resulting in a one-dimensional function described by equation 4

$$
S_{E E S}(\alpha)=\frac{1}{f_{s} / 2} \int_{0}^{f_{s} / 2}|\gamma(\alpha, f)| d f
$$

where $f_{s}$ is the Nyquist frequency. For numerical implementation, equation 4 is replaced by discrete sums over the spectral frequency $f$.

When the $\gamma(\alpha, f)$ matrix is integrated along the spectral frequency dimension, the result is one dimensional vector function of the cyclic frequency $\alpha$ [15. 
As can be implied from the equations, the $\operatorname{EES}\left(S_{E E S}(\alpha)\right)$ is similar to the SES, as it represents the spectrum of the demodulated signal, where the modulation frequencies present in the raw signal are exhibited. The EES is expected to provide a better enhancement of the cyclic components when compared against the SES.

When the integration of the CSCoh is performed along the full ensemble of the frequency lines $\left[0 ; f_{s} / 2\right]$, high noise level may remain the EES. Instead, the CSCoh can be integrated over a small band on the spectral frequency. This is analogous to "band-pass filtering" before the signal demodulation, because only the cyclic components with the specific carrier frequencies are present in the spectrum in the end. As can be implied, application of this idea is akin to the Envelope Analysis, where the high frequency band that contains the fault content is selected and is "filtered". In this case the high frequency content along the cyclic frequency $\alpha$ containing the fault frequencies information is selected, and the integration of the CSCoh occurs over the correspondent $\left[f_{1} ; f_{2}\right]$ band of frequency. The resulting spectrum, coined Improved Envelope Spectrum (IES) [16], is thus obtained with enhanced fault frequencies, and described by equation 5.

$$
S_{I E S}(\alpha)=\frac{1}{f_{2}-f_{1}} \int_{f_{1}}^{f_{2}}|\gamma(\alpha, f)| d f
$$

It can be noticed that the corresponding spectrum after the integration on the CSCoh can be either termed EES or IES, if the integration is realised over all the frequency band $\left[0 ; f_{s} / 2\right]$ or on a selected band $\left[f_{1} ; f_{2}\right]$. This distinction is made because the EES is equivalent to the pure signal enveloping, whilst the IES is equivalent to filtering the signal before demodulation (i.e. Envelope Analysis).

The IES allows improvement of the damage diagnosis when compared to EES, even more on signals with strong components that mask the fault information. Thus it is highly compatible with this paper stated problem, where EMI contamination mask the bearing fault detection. On the other hand, the 
diagnosis is dependant on the selection of the correct band to integrate on the CSCoh, leading to true diagnosis of the fault.

The specific band that optimizes the fault frequencies components on the resulting IES can be extracted by analysis of the CSCoh, much like analysis of the raw spectrum allows the selection of the band-pass filter parameters for the filtered SES from the Envelope Analysis technique, thus making the procedure time consuming while requiring experience. Another solution would be to use a band selection tool like the Kurtogram, but no universal method with application on the CSCoh has been implement yet. This maybe due to implementation of the CSC based methods being fairly recent as condition monitoring tools.

\section{New diagnostic approaches based on the integration of the Cyclic Spectral Coherence}

In this paper two new approaches for automatic selection of the integration corresponds to the "IESFOgram or IESAM criterion" box.

\subsection{Improved Envelope Spectrum by Alpha Maximization (IESAM)}

The first criterion detects the band of frequencies that maximize the spectral frequency power along the alpha lines equal to the fault frequencies $\left(f_{\text {fault }}\right)$ and their first $k^{t h}$ harmonics, $\alpha_{k}=k f_{\text {fault }}$, thus the name Improved Envelope Spectrum by Alpha Maximization.

Assuming spectral carrier frequencies are excited, these frequencies are exhibited in the CSCoh as local maxima at each cyclic fault frequency. In 


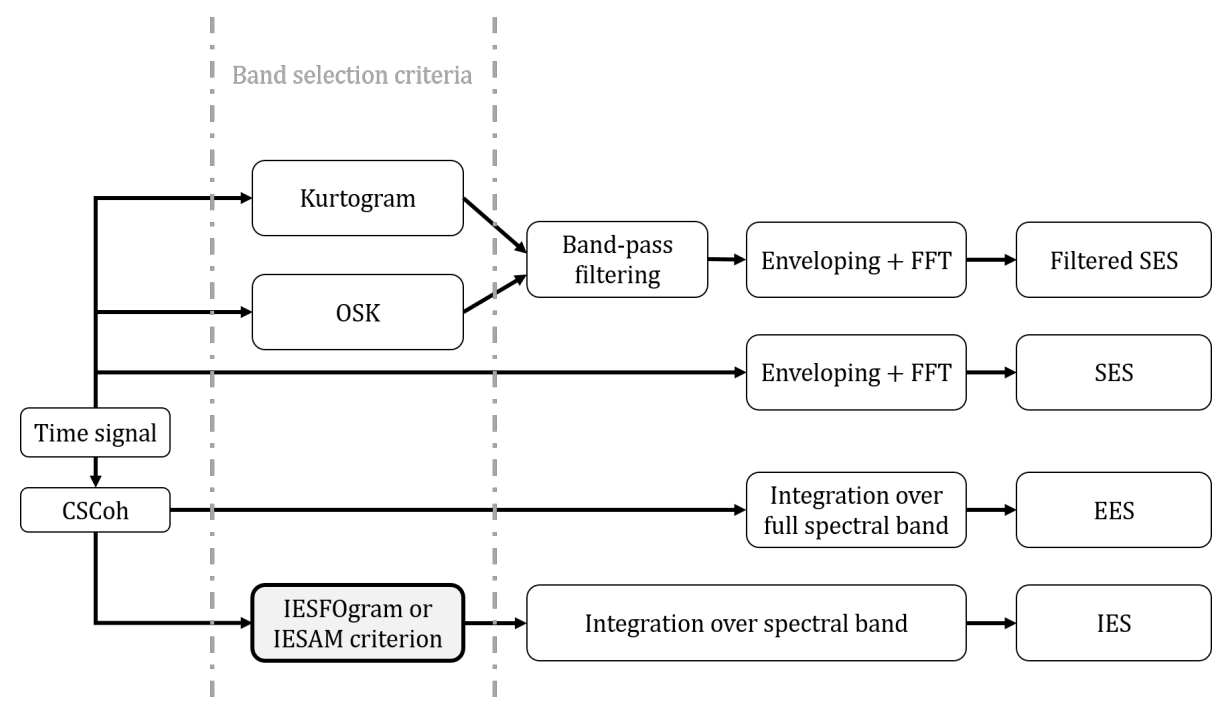

Figure 5: Schematic description of EA, classical SES, EES and IES.

other words, the carrier frequency can be extracted from these local maxima when a cyclic fault frequency is present. Naturally, this band can be selected as the band of frequencies that maximizes the amplitude of the frequencies along the demodulated harmonics of said fault frequency.

From the CSCoh matrix $\gamma_{x}(\alpha, f)$, the vectors along the first $k$ harmonics of the cyclic fault frequency are obtained, resulting in $k$ vectors $\gamma_{\alpha_{k}}$ function of spectral frequency $f$. After this, the inner product of all the $\gamma_{\alpha_{k}}$ vectors is calculated according to the equation 6

$$
\gamma_{A M}(f)=\left\langle\gamma_{\alpha_{1}}(f), \gamma_{\alpha_{2}}(f), \quad \ldots \quad, \gamma_{\alpha_{i}}(f)\right\rangle, \quad \alpha_{i}=i \cdot f_{\text {fault }}
$$

The final vector $\gamma_{A M}(f)$ is expected to declare the range of spectral frequency 318 $f$ where the fault content is dominant. Defining a threshold thres (e.g. thres $=70 \%$ of the maximum), allows the selection of a band around this maximum, and integrating $f$ over this band results in the IESAM that contains the fault frequencies. The procedure for obtaining the criterion for the band selection of the IESAM is exemplified in figure 6.

The criterion to select the optimal band is not blind. This means the 


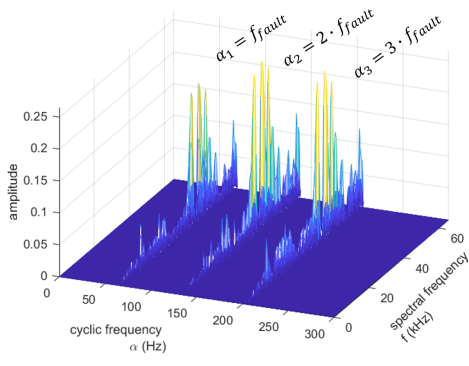

(a) Fault $\alpha$ frequencies on CSCoh.

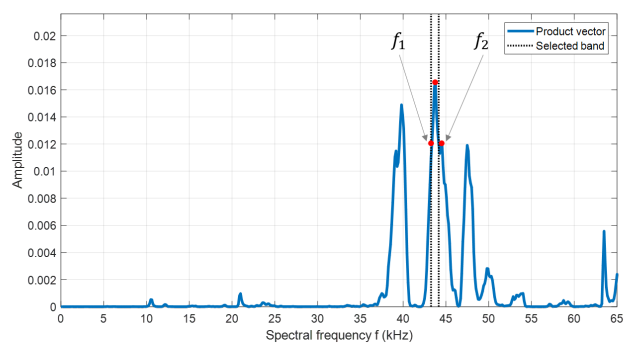

(b) Criterion for the IESAM band selection.

Figure 6: Example on the procedure to obtain the criterion for the IESAM band selection by the inner product of three vectors from three harmonics of the fault frequency.

324 step, the first $k$ harmonics of the fault frequency along the cyclic frequency $\alpha$ axis of the CSCoh are obtained. This is performed by searching a small range around the fault frequency and the maximum value returns the real cyclic frequency of the fault. The three spectral frequency lines corresponding to these cyclic frequencies are then obtained. These $k$ vectors are then multiplied, highlighting results with this criterion on this paper apply a threshold of $70 \%$ around the maximum of this vector to select the range between the lower frequency $f_{1}$ and the upper frequency $f_{2}$. The final step is to integrate the CSCoh on the selected rage $\left[f_{1} ; f_{2}\right]$ over the spectral frequency in order to obtain the IESAM (the IES selected by the IESAM criterion). The steps to perform the IESAM are described in the flowchart of figure 7

\subsection{Improved Envelope via Feature Optimization -gram (IESFOgram)}

As the name suggests, the Improved Envelope Spectrum via Feature Optimization (IESFOgram) attempts to optimize the amplitude of a predefined frequency feature from the integration of a specific band of the CSCoh. In order to provide a solid comparison with IESAM, this paper selected the feature 


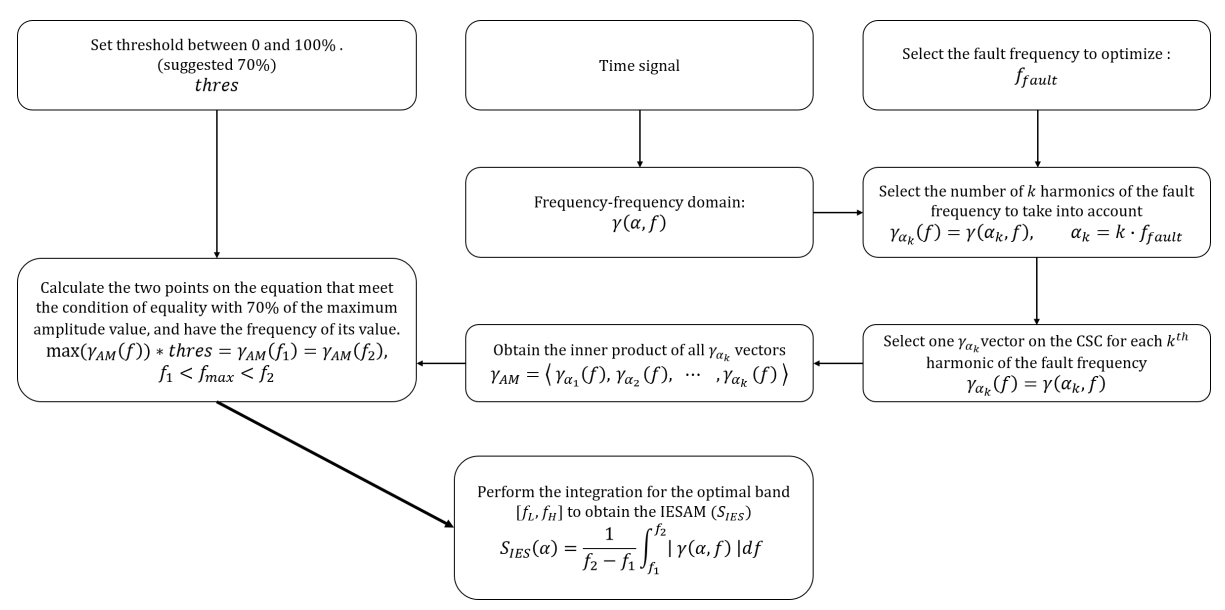

Figure 7: Flowchart of the proposed method IESAM.

characteristic bearing fault frequency. This feature can be expressed by equation 7

$$
\text { Ind }=\sum_{i=1}^{k} S_{I E S}\left(\alpha_{i}=i \cdot f_{\text {fault }}\right)
$$

where $f_{\text {fault }}$ is the characteristic bearing fault frequency, and $k$ the number of harmonics to take into account.

This feature is based on the specific bearing frequency, however nothing impedes the selection of a different feature. For example, a combination of all characteristic frequencies of a bearing can be extracted for diagnosis targeted to a specific bearing, or, frequency domain statistics can be extracted for blind analysis. Time domain features are not compatible with the method.

It is similar to FK, where a series of bands along the spectral frequency axis $f$ with different bandwidths $b w$ and center frequencies $c f$ are selected in 354 a $1 / 3$ binary tree. The IES $S_{I E S}$ for each combination of $(b w, c f)$ is obtained by integration around the center frequency $c f$ spaced by the bandwidth $b w$. Starting from the full band from $\mathrm{OHz}$ up to half the Nyquist frequency, the bandwidths decrease for each increasing order of the binary tree. For each $S_{I E S}$, the feature Ind is estimated. Finally a 2D map is created, just like FK, where 
the Ind is plotted as a function of the center frequency and the order (which is a logarithmic relation with the size of the bandwidth). This 2D map binary tree is the selection criterion to obtain the IESFO, and as it is represented by the same visualization as the Kurtogram, being coined as the IESFOgram. In other words, the highest feature value on this IESFOgram corresponds to the optimal band. Integration of the CSCoh on this band results then on the IESFO, which should exhibit clearly high amplitude values on the fault frequencies, assuming the selected bearing fault is present in the signals.

Smith et. al. also proposed a criterion which maximizes the secondorder cyclostatonarity at the bearing fault, with is the same definition of the IESFOgram, but applied to the filtered SES instead. The criterion provided is ICSgram, and as the name suggests, it has the same visualization of the Kurtogram and IESFOgram [17. The ICSgram showed better performance than the Kurtogram, but as it is only applied to the SES, it is expected the IESFOgram to have better performance due to CSCoh being more sensitive to weak cyclostationary signals.

A flowchart showing the implementation of the IESFOgram procedure is shown in figure 8 .

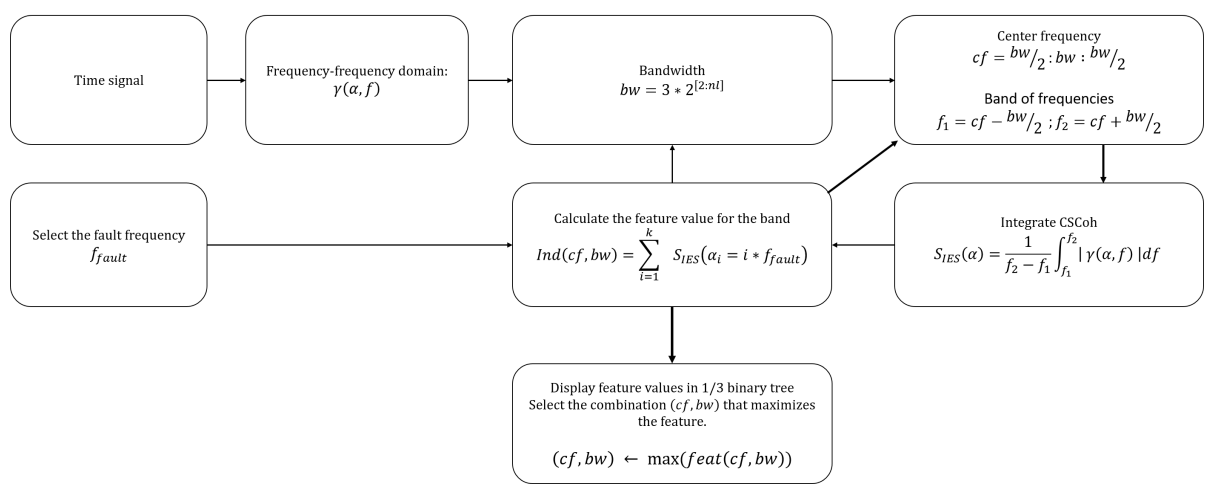

Figure 8: Flowchart of the proposed method IESFO. 


\section{Experimental Setup}

378 depicted in figure 9. A three-phase induction motor drives a hydraulic system to provide torque to the gearbox, while the input shaft speed is controlled by the VFD [2]. The gearbox has one parallel and one planetary stage. The ring gear is fixed, while the planet carrier is the input to the planetary stage and the sun gear the output. The description of the gearbox reduction stages are described in figure 9. An accelerometer is mounted on the planet carrier to measure acceleration in the axial direction, to investigate the use of internal vibration measurements, alongside one slip ring which is used to transmit the vibration signal.

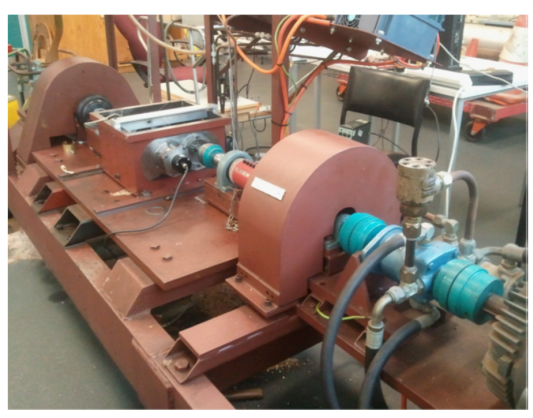

(a) Test rig.

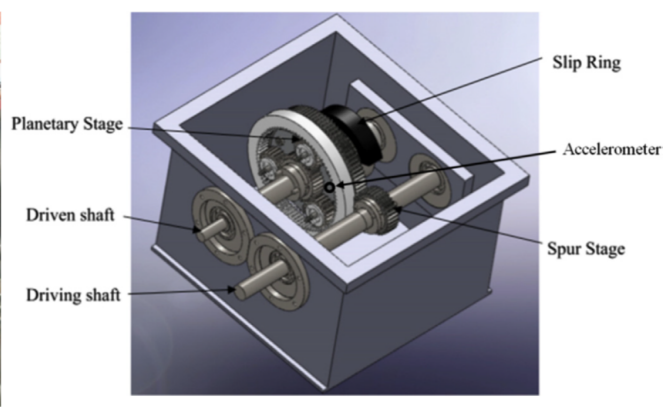

(b) CAD representation.

Figure 9: Planetary gearbox.

All the measurements were taken under constant speed of $6 \mathrm{~Hz}$. The carrier gear rotates at $2 \mathrm{~Hz}$, as it meshes with the gears of the input shaft with a initial reduction ratio of 90:32. Faults have been seeded in the inner race and the outer races of the planet gear bearings using spark erosion. Needle roller bearings are used, containing 15 rollers of $2 \mathrm{~mm}$ diameters and a pitch diameter of $18 \mathrm{~mm}$. The depth of the faults is $0.4 \mathrm{~mm}$ while the width is $1.2 \mathrm{~mm}$ for the outer race and $1.0 \mathrm{~mm}$ for the inner race. One of the planet gears contain the faulty bearing, while the ring gear is fixed. The outer race fault frequency (BPFO) is about $56 \mathrm{~Hz}$, and the inner race fault frequency (BPFI) is about $69 \mathrm{~Hz}$. Testing 
was conducted with three braking loads of 30,50 and $70 \mathrm{Nm}$ for each type of defect (inner and outer race). The sampling frequency is 131,072 Hz, the switching frequency of the VFD is set at $14 \mathrm{kHz}$, and the control frequency of the VFD is $24 \mathrm{~Hz}$ (giving a nominal constant $6 \mathrm{~Hz}$ shaft speed for the 8-pole motor) [2]. listed in table 1 .

Table 1: List of expected frequencies

\begin{tabular}{ll} 
Expected frequencies & Value $[\mathbf{H z}]$ \\
\hline PWM carrier $\left(f_{c}\right)$ & 14000 \\
PWM message $\left(f_{m}\right)$ & 24 \\
Current supply $($ mains $)$ & 50 \\
2nd current supply & 100 \\
Ball pass fault frequency of outer race ( BPFO ) & 55 \\
Ball pass fault frequency of inner race ( BPFI $)$ & 69 \\
Planet carrier ( $\left.f_{p}\right)$ & 2 \\
Gear mesh frequency $(G M F)$ & 165
\end{tabular}

For the analysis of this dataset, the number of fault frequency harmonics considered is $k=3$. In other words, with the signals containing inner race faults, 3 harmonics of the BPFI are considered. As for the outer race fault signals, 3 harmonics of the BPFO are also taken into account. This selection is applied to all the described methods, whose results are discussed in the next section.

\section{Results and Discussion}

Figure 10 shows the envelope spectra obtained using the classical EES and SES approaches for the inner race fault and maximum load $(70 \mathrm{Nm})$ case. The EES gives a reasonably clear diagnosis, with strong harmonics of BPFI, ${ }_{414}$ but with a dominant component at $100 \mathrm{~Hz}$ (twice mains frequency). The 
classical SES without filtering shows no results in diagnosing the faults, as the BPFI is completely masked by the discrete gearbox frequencies and the EMI components. The outer race fault is not shown as the results are similar to the inner race fault with $70 \mathrm{Nm}$ braking load, with detection using on the EES and no detection on the SES.

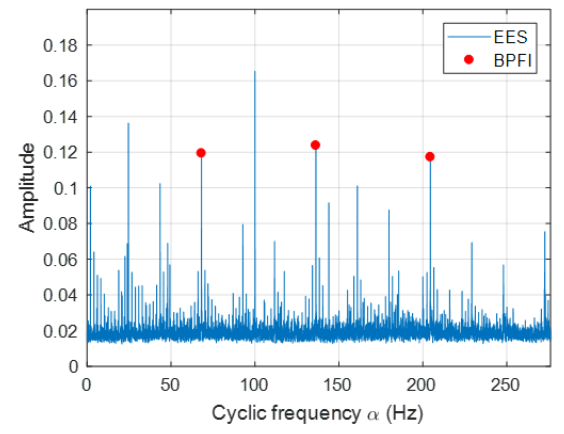

(a) Classical EES.

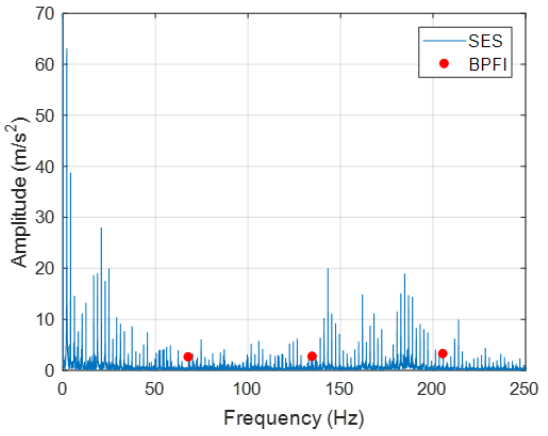

(b) Classical SES.

Figure 10: Spectra of inner race fault with breaking load of $70 \mathrm{Nm}$.

From this case of $70 \mathrm{Nm}$ the advantage of the classical EES when compared to the classical SES becomes apparent as it shows better results in enhancing the fault frequencies. However, when the load is decreased, the EES no longer detects clearly the fault frequencies, and further processing is needed. This is likely due to the fault impulses decreasing with the load, and thus becoming increasingly masked in the EMI. In figure 11 it can be seen the BPFI is no longer evident with the classical EES for the 50 and $30 \mathrm{Nm}$ cases.

After analysing the different filters along the full band of the spectrum in order to obtain a Filtered SES, the analysis showed that the bands that give a clear fault diagnosis are centred around the $3^{\text {rd }}$ harmonic of the PWM carrier frequency, which is around $42 \mathrm{kHz}$. The boxes in figure show the optimal bands of demodulation that enhance the fault frequencies, for both inner and outer race faults. Note in figure 12 that the optimal bands avoid the $3^{\text {rd }}$ harmonic of the carrier of the PWM, at $42 \mathrm{kHz}$, where the fault frequencies are present, but the spectrum is dominated by the EMI. 


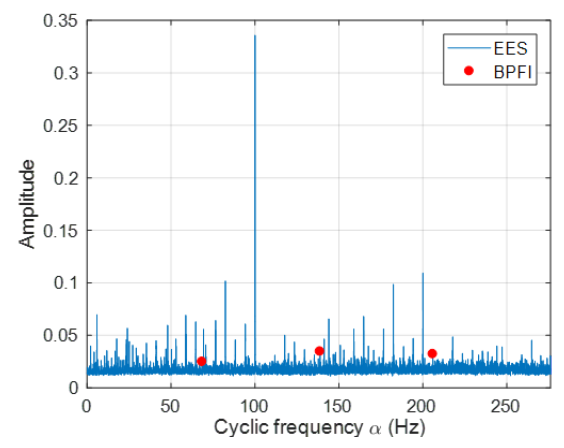

(a) $30 \mathrm{Nm}$ load.

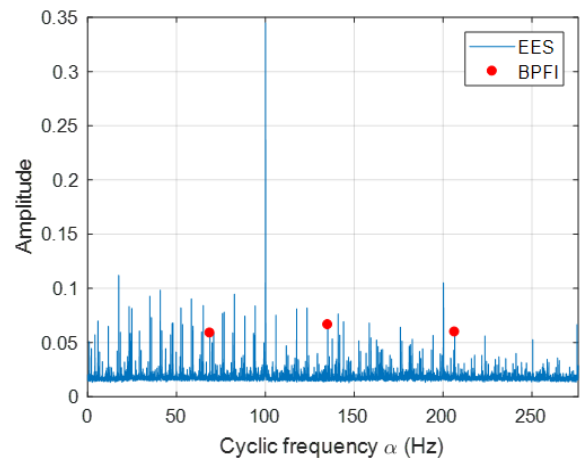

(b) $50 \mathrm{Nm}$ load.

Figure 11: EES with inner race fault under two torque loads.

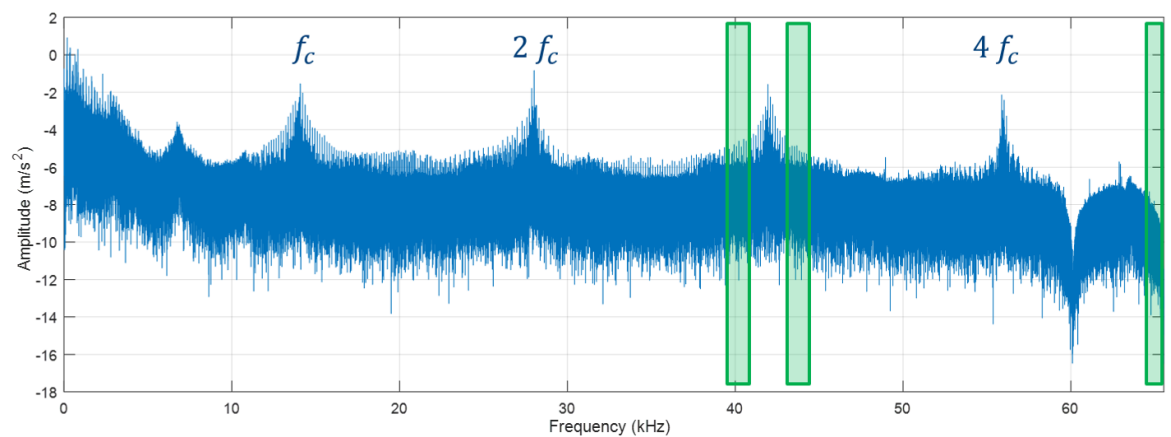

Figure 12: Raw spectrum showing the carrier frequencies of the EMI and the correct band filters (green) around the EMI $3^{\text {rd }}$ harmonic and at the Nyquist.

Applying bandpass filters centred around these frequencies, the spectra in figure 13 confirms that a filter around $44 \mathrm{kHz}$ results in clear peaks at the fault frequencies. On the other hand, filtering exactly on the $3^{\text {rd }}$ harmonic of the PWM carrier, $42 \mathrm{kHz}$, the BPFI harmonics are still present, but the EMI related frequencies are dominant.

In fact, the OSK results provide a clear fault detection for all loads and for both inner and outer race faults exactly because the method always select one of these three bands, while avoiding the exact $3^{\text {rd }}$ harmonic of the carrier. Applying one of the methods proposed by this paper, the IESAM criterion also 


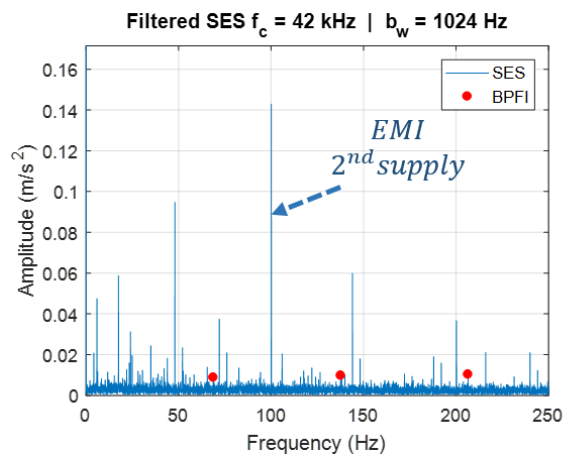

(a) $c f$ at the $3^{r d} f_{c}$.

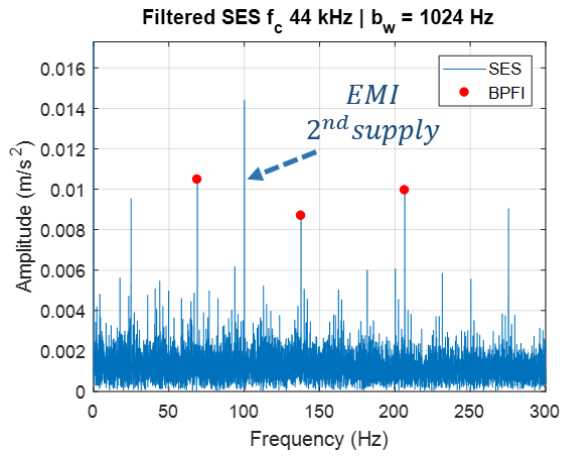

(b) $c f$ near the $3^{r d} f_{c}$ EMI harmonic.

Figure 13: Filtered SES around a center frequency $c f$.

444

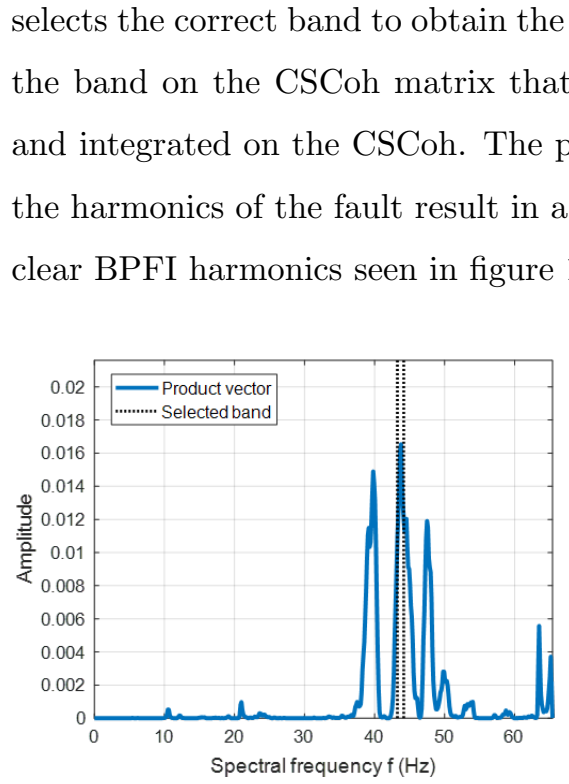

(a) IESAM criterion for band selection.

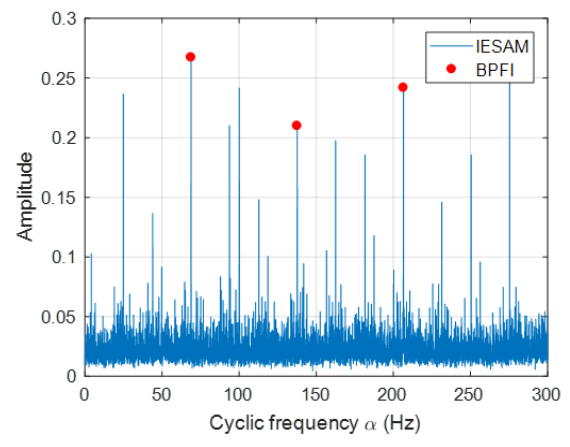

(b) Resulting IESAM.

Figure 14: IESAM method applied to inner race fault with load $50 \mathrm{Nm}$.

Application of IESAM method to the $70 \mathrm{Nm}$ and to the $30 \mathrm{Nm}$ cases also results in the selection of an optimal band for integration of the CSCoh, thus clear detection of the fault frequencies for all the cases is obtained using this method. 
Applying the second proposed method, it can be seen in figure 15 the IESFOgram shows the two largest feature values near $42 \mathrm{kHz}$, similar to the IESAM criterion result. Both of these bands result in clear fault frequency components, and for the case of $50 \mathrm{Nm}$ as the maximum is at $44 \mathrm{kHz}$, this was the selected center frequency. The IESFOgram also shows that the feature value increases with narrower bandwidths, where the maximum occurs on order 6 , resulting in a bandwidth of $341 \mathrm{~Hz}$. The integration of this band results in clear fault frequency peaks on the spectrum.

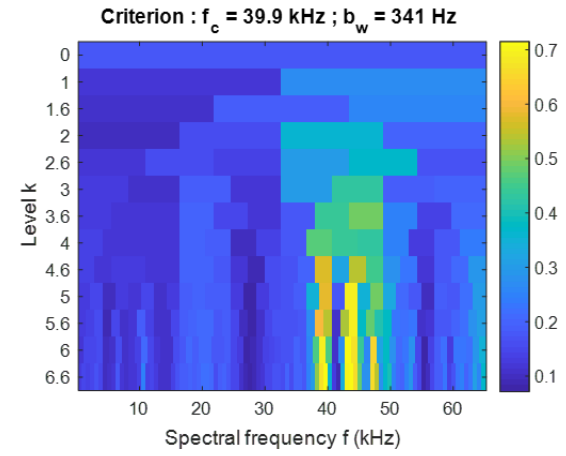

(a) IESFOgram.

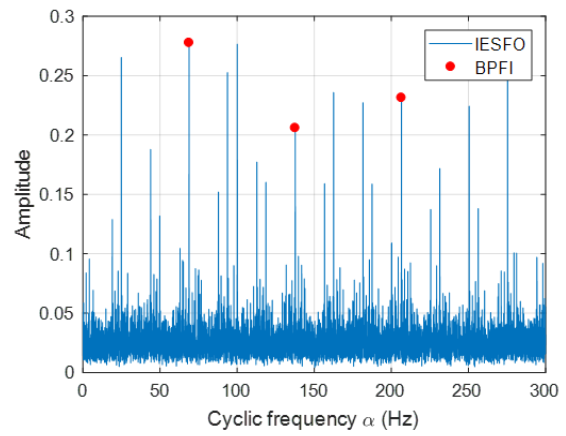

(b) Resulting IESFO.

Figure 15: IESFO method applied to inner race fault with load $50 \mathrm{Nm}$.

The IESFO also provides a clear detection of the fault frequencies for the other cases, with loads $30 \mathrm{Nm}$ and $70 \mathrm{Nm}$, as well as for the outer race fault case.

The manual analysis of the signals showed that, when the Signal-to-Noise Ratio of the fault content was low, the EMI related frequencies were the highest in the spectra. As such, an evaluation indicator (Feature) is introduced in this paper as a means to represent the diagnosis performance of the various methods and whether the fault is clearly detected.

The indicator is defined as the ratio between the sum of the first $k$ fault harmonics ( $k=3$ used here) and the maximum value on the spectrum. The indicator is calculated as shown below in equation 8 


$$
\text { Feature }=\frac{2}{k} \frac{\sum_{i=1}^{k} A\left(f=i \cdot f_{\text {fault }}\right)}{\max (A(f))}, \quad k=1,2, \ldots, N
$$

where $k$ is the number of harmonics of the fault, and $A(f)$ is the amplitude at a frequency $f$ on the demodulated spectrum (SES, EES, IES). The parameter equal to 1

Figure 16 demonstrates the feature for all the methods mentioned in this paper.

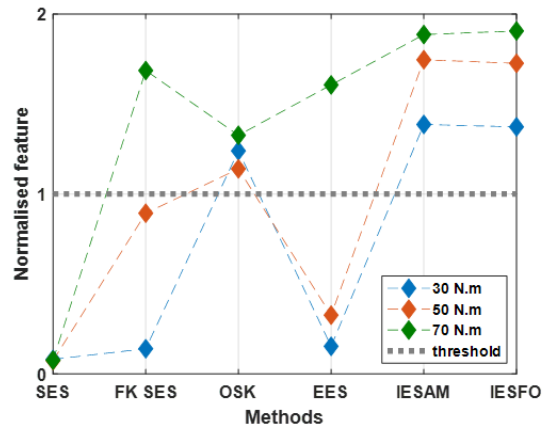

(a) Inner race fault features.

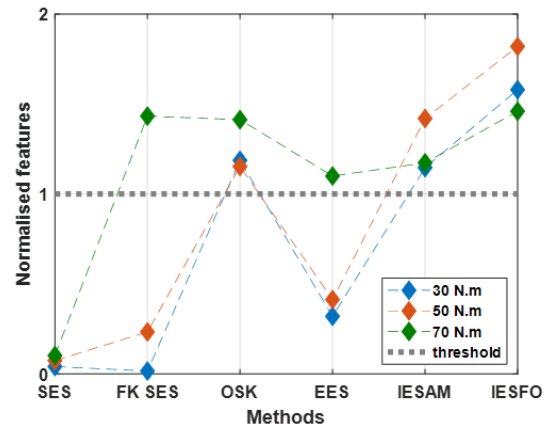

(b) Outer race fault features.

Figure 16: Feature for all methods and loads for the inner race fault and the outer race fault.

Based on the evaluation indicator and confirmed through visual analysis of the spectra, the bearing faults are identified for all load cases for both the proposed methods (IESAM and IESFO), demonstrating the effectiveness of band selection for the IESFOgram and IESAM criterion. 


\section{Conclusion}

In this paper two novel tools, entitled Improved Envelope Spectrum by Alpha Maximitixation criterion (IESAM criterion) and Improved Envelope Spectrum via Feature Optimization-gram (IESFOgram), have been proposed focused towards bearing diagnostics under strong Electromagnetic Interference (EMI). The tools provide an automated selection of the optimal band of integration on the the Cyclic Spectral Coherence (CSCoh) or the Cyclic Spectral Correlation (CSC), leading to the estimation of the Improved Envelope Spectrum (IES).

The IESFOgram is visual tool similar to the Kurtogram, which is measures the cyclostationarity level of a characteristic fault frequency, and selects the parameters which correspond to the maximum level. The IESAM criterion produces a 1-D function that describes the spectral density for a specific fault frequency, and selects the optimal band based on the maximum of this function.

Two methodologies, leading to the IES by application of each of the two criteria respectively, are evaluated on bearing signals captured over a planetary gearbox under strong EMI influence. The methodologies have been further compared to the Kurtogram based Squared Envelope Spectrum (SES), the Optimised Spectral Kurtosis (OSK) based SES, and the classical Enhanced Envelope Spectrum approach.

Both proposed methodologies resulted on the successful detection of the outer race and inner race fault of the planet gear bearings, for all cases. Based on this results, the integration of the CSCoh on the band selected by either the IESFOgram or the IESAM criterion appear to be a powerful tool in detecting masked bearing faults in planetary gearbox under strong EMI noise.

The methods good performance in detecting various faults also suggest that their applicability could go beyond EMI contaminated signals and bearing faults into more general condition monitoring challenges. 


\section{Acknowledgment}

516 gratefully acknowledge Flanders Make and VLAIO Flanders Innovation and Entrepreneurship Agency within the VIBMON project for their support. K Gryllias gratefully acknowledges the Research Fund KU Leuven.

\section{References}

[1] W. A. Smith, R. B. Randall, Rolling element bearing diagnostics using the case western reserve university data: A benchmark study, Mechanical Systems and Signal Processing 64 (2015) 100-131.

[2] W. Smith, Z. Fan, Z. Peng, H. Li, R. Randall, Optimised spectral kurtosis for bearing diagnostics under electromagnetic interference, Mechanical Systems and Signal Processing 75 (2016) 371-394.

[3] W. A. Smith, Z. Fan, D. Peng, Z. Peng, R. B. Randall, Electromagnetic interference in vibration signals and its effect on bearing diagnostics, in: Proceedings of Vibrations in Rotating Machinery - VIRM11, Academic Press, 2016, pp. 01-11.

[4] J. Antoni, Fast computation of the kurtogram for the detection of transient faults, Mechanical Systems and Signal Processing 21 (1) (2007) 108 - 124.

[5] R. Randall, J. Antoni, Rolling element bearing diagnostics - a tutorial, Mechanical Systems and Signal Processing 25 (2011) 485-520.

[6] K. Gryllias, I. Antoniadis, A peak energy criterion (pe) for the selection of resonance bands in complex shifted morlet wavelet (csmw) based demodulation of defective rolling element bearings vibration response, International Journal of Wavelets, Multiresolution and Information Processing 7 (4) (2009) $387-410$. 
[7] N. Sawalhi, R. B. Randall, The application of spectral kurtosis to bearing diagnostics, in: Proceedings of Acoustics 2004, Academic Press, 2004, pp. 393-398.

[8] T. Wang, Q. Han, F. Chu, Z. Feng, A new skrgram based demodulation technique for planet bearing fault detection, Journal of Sound and Vibration 385 (2016) $330-349$.

546 [9] J. Antoni, The infogram: Entropic evidence of the signature of repetitive transients, Mechanical Systems and Signal Processing 74 (2016) 73-94.

[10] A. Mauricio, C. Freitas, J.Cuenca, B. Cornelis, K. Janssens, K. Hendrickx, K. Gryllias, Condition monitoring of gears under medium rotational speed, in: International Congress on Sound and Vibration- ICSV24, International Institute of Acoustics and Vibration, 2017, pp. 01-08.

552 [11] J. Antoni, G. Xin, N. Hamzaoui, Fast computation of the spectral correlation, Mechanical Systems and Signal Processing 92 (2017) 248 277.

[12] J. Antoni, Cyclic spectral analysis in practice, Mechanical Systems and Signal Processing 21 (2) (2007) $597-630$.

[13] P. McFadden, J. Smith, Model for the vibration produced by a single point defect in a rolling element bearing, Journal of Sound and Vibration 96 (1) (1984) $69-82$.

[14] D. Abboud, S. Baudin, J. Antoni, D. Rmond, M. Eltabach, O. Sauvage, The spectral analysis of cyclo-non-stationary signals, Mechanical Systems and Signal Processing 75 (Supplement C) (2016) 280 - 300.

[15] J. Antoni, Cyclostationarity by examples, Mechanical Systems and Signal Processing 23 (4) (2009) 987 - 1036.

[16] D. Abboud, J. Antoni, Order-frequency analysis of machine signals, Mechanical Systems and Signal Processing 87 (2017) 229 - 258. 
[17] W. A. Smith, R. Randall, X. de Chasteigner du Me, Z. Peng, Use of cyclostationary properties to diagnose planet bearing faults in variable speed conditions, in: Tenth DST Group International Conference on Health $570 \quad$ and Usage Monitoring Systems (HUMS), Academic Press, 2017, pp. 01-11. 